\title{
MECHANISM OF INTRINSIC FACTOR ACTION IN EVERTED SACS OF RAT SMALL INTESTINE*
}

\author{
By VICTOR HERBERT $\dagger$ \\ (From the Department of Hematology, Division of Laboratories, Montefiore Hospital, \\ New York, N. Y.)
}

(Submitted for publication July 28, 1958; accepted September 18, 1958)

Three decades have passed since Castle (1) and his associates demonstrated the existence of intrinsic factor, yet knowledge of its mechanism of action is still so limited that in 1958 two workers felt justified in concluding there was no such thing as intrinsic factor (2). In prior studies $(3,4)$, a mechanism involved in the enhanced uptake of vitamin $B_{12}$ by rat liver slices in the presence of hog intrinsic factor concentrate (HIFC) was demonstrated. This mechanism appeared to be dependent on the presence on liver slices of receptors for intrinsic factor, which in the presence of calcium would "take up" nonmetabolically either free intrinsic factor or intrinsic factor bound to vitamin $\mathrm{B}_{12}$. The present studies indicate the same mechanism may operate in the rat small intestine. A preliminary report of this work has been presented (5).

\section{MATERIALS AND METHODS}

The basic protocol for each experiment was that previously used in studies with rat liver slices (4). It consisted of two successive one hour incubations of everted sacs (6) of rat small intestine at $6^{\circ} \mathrm{C}$. in air; the first with $0.5 \mathrm{mg}$. HIFC ${ }^{1}$ in the Krebs-Ringer-Tris (4) incubation medium, the second with $0.01 \mu \mathrm{g}$. vitamin $\mathrm{B}_{12}-\mathrm{Co}^{80}$ added. Controls were run identically, except for exclusion of HIFC. Radioactivity retained in the mucosa of the everted sacs after three washings was determined in a well-type scintillation counter. Each sample was counted

* Supported in part by Grant H-3461 from the National Institutes of Health, United States Public Health Service, and in part by a grant from the New York Heart Association and Heart Fund, Inc.

† Senior Research Fellow, New York Heart Association and Heart Fund, Inc. Present address: Department of Hematology, The Mount Sinai Hospital, New York, N. Y.

1 Kindly provided by Dr. W. L. Williams and Dr. L. Ellenbogen, Lederle Laboratories, American Cyanamid Company, Pearl River, N. Y. This preparation (No. WES671A) was clinically active in a daily oral dose of 5 mg., as determined by urinary excretion studies of the Schilling type (7). for a minimum of 10,000 counts. Radioactivity was expressed as counts per minute per $10 \mathrm{~cm}$. of small intestine length.

The sacs were prepared as follows: A healthy adult female rat of the Wistar strain was sacrificed. The small intestine was immediately removed by transecting the gut at the duodenal end, gently pulling it free of its mesenteric attachment, and transecting it again at the distal end of the ileum. The proximal $6 \mathrm{~cm}$. was discarded. A No. 6 Cournand single lumen woven cardiac catheter ${ }^{2}$ was slipped through the lumen of the small intestine, starting at the ileal end. When the catheter tip appeared at the duodenal end, a ligature was placed tightly over the ileal end to prevent passage of the base of the catheter. Continuous gentle traction on the tip of the catheter then brought about complete eversion of the small intestine, which was immediately placed in 0.9 per cent $\mathrm{NaCl}$ at $0^{\circ}$ C. Adherent intestinal content was removed by washing in three changes of ice-cold 0.9 per cent $\mathrm{NaCl}$. Starting at the duodenal end, the intestine was cut into $20 \mathrm{seg}$ ments of equal length. The ends of each segment were closed with Schwartz straight temporary arterial intracranial clamps. ${ }^{3}$ No detectable adsorption of vitamin $\mathrm{B}_{12}-\mathrm{Co}^{\infty 0}$ onto these clamps was noted after the usual washings. The segments were placed sequentially into four beakers containing the standard incubation medium, so that segments numbered $1,5,9,13$ and 17 were in beaker No. 1, and so forth. Although only one experiment of each type is reported, each experiment was repeated at least twice for verification.

\section{RESULTS}

Simultaneous incubation. Sacs incubated in the standard medium with both HIFC and vitamin $\mathrm{B}_{12}-\mathrm{Co}^{80}$ added simultaneously showed no enhancement of vitamin $B_{12}$-uptake due to HIFC (Experiments 1 and 2, Table I). This was true at six different concentrations of HIFC, including the concentration $(25 \mu \mathrm{g}$.) which produced a more than threefold enhancement of vitamin $\mathrm{B}_{12}-\mathrm{Co}^{60}$ uptake in the simultaneous incubation system with rat liver slices (4).

\footnotetext{
2 Purchased from United States Catheter and Instrument Corp., 334 Bay Street, Glen Falls, N. Y.

3 Purchased from Storz Instrument Co., 4570 Audubon Avenue, St. Louis 10, Mo.
} 
TABLE I

Effect of hog intrinsic factor concentrate $(H I F C)$ on vitamin $B_{12}-C_{0}^{60}$ uptake by everted sacs of rat small intestine under various experimental conditions

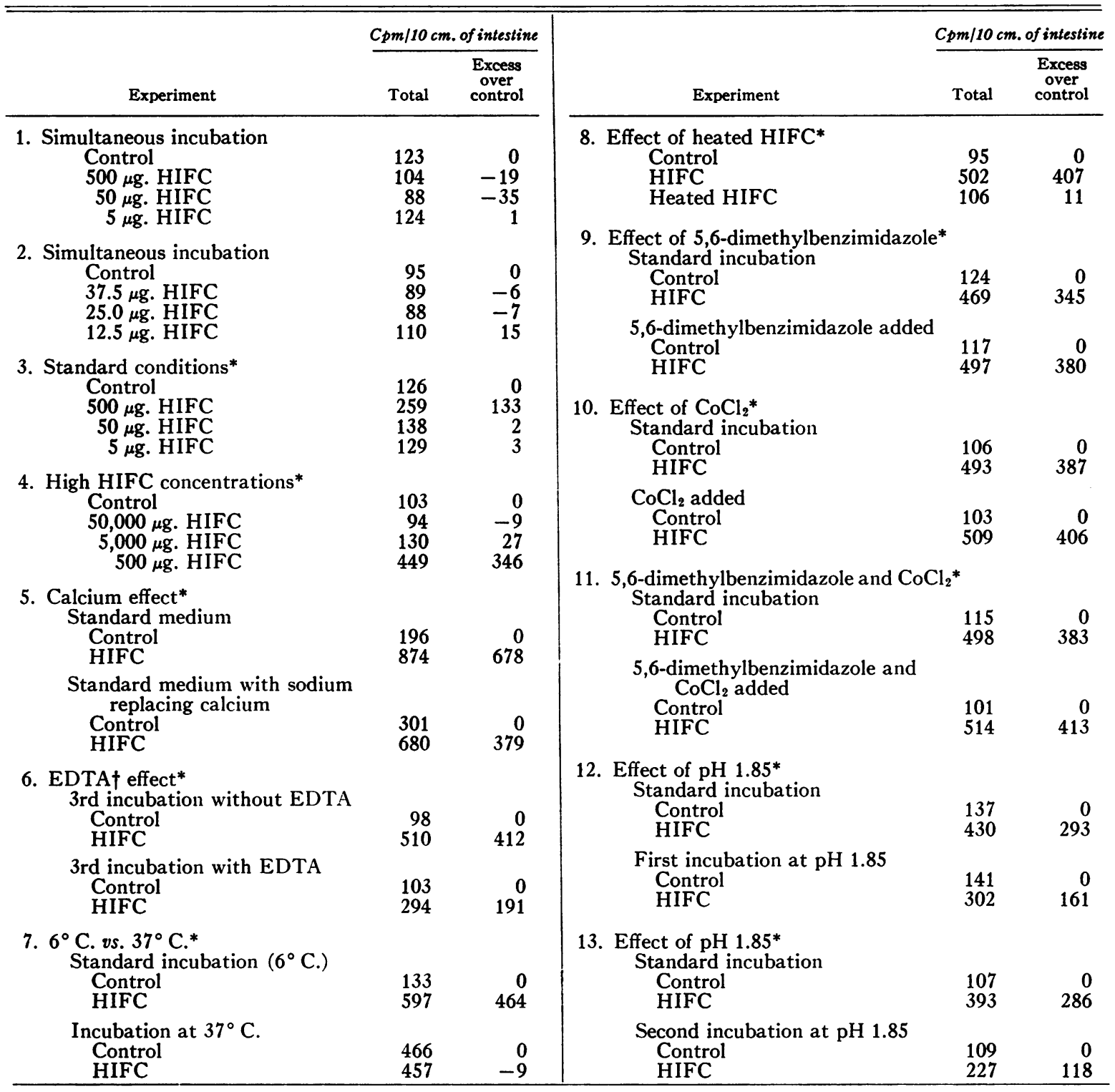

* Sequential incubation, i.e., two successive one-hour incubations: the first with $0.5 \mathrm{mg}$. HIFC (unless otherwise stated) in the incubation medium, the second with $0.01 \mu \mathrm{g}$. vitamin $\mathrm{B}_{12}-\mathrm{Co}^{60}$ added.

$\dagger$ Disodium ethylenediaminetetraacetate dihydrate.

Sequential incubation. Sacs incubated first with $0.5 \mathrm{mg}$. of $\mathrm{HIFC}$ and then with vitamin $\mathrm{B}_{12}-\mathrm{Co}^{60}$ invariably showed a significant enhancement of the uptake of vitamin $\mathrm{B}_{12}-\mathrm{Co}^{60}$ (Table I). However, no clear enhancement was observed in the sequential incubation system when 10 -fold or 100 -fold higher or lower concen- trations of HIFC were used (Experiments 3 and 4, Table I).

Effect of calcium-free medium. The enhancement of uptake produced by HIFC was markedly reduced in the absence of calcium (Experiment 5, Table I). The marked increase in the uptake of vitamin $\mathrm{B}_{12}-\mathrm{Co}^{60}$ by control tissues in the 
absence of calcium may be due to increased cell permeability. The same phenomenon has been observed in studies with rat liver slices (3).

Effect of disodium ethylenediaminetetraacetate dihydrate $(E D T A)$. After performance of the standard experimental procedure, sacs were incubated for a third hour in $10 \mathrm{ml}$. of KrebsRinger-Tris to which was added $2 \mathrm{ml}$. of 0.9 per cent $\mathrm{NaCl}$ either without disodium ethylenediaminetetraacetate dihydrate (EDTA) or containing $2 \mathrm{Gm}$. of the material per $100 \mathrm{ml}$. As noted in Table I, Experiment 6, the addition of EDTA brought about no change in control values, but markedly reduced the amount of vitamin $\mathrm{B}_{12}-\mathrm{Co}^{60}$ retained by sacs previously incubated in the presence of added HIFC.

Temperature. When the standard two incubations were performed at $37^{\circ} \mathrm{C}$. instead of $6^{\circ} \mathrm{C}$., the intestinal sac mucosa became pale and friable; microscopic examination ${ }^{4}$ demonstrated destruction of the mucosa. Such sacs showed very high control uptake and no enhancement by HIFC (Experiment 7, Table I).

Heating HIFC. The addition of HIFC which had been heated in a water bath at $100^{\circ} \mathrm{C}$. for 15 minutes resulted in no enhancement of uptake of vitamin $\mathrm{B}_{12}-\mathrm{Co}^{60}$ (Experiment 8, Table I).

Effect of 5,6-dimethylbenzimidazole ${ }^{5}$ and $\mathrm{CoCl}_{2}$. Neither 5,6-dimethylbenzimidazole nor $\mathrm{CoCl}_{2}$ interfered with the uptake of vitamin $\mathrm{B}_{12}-\mathrm{Co}^{60}$ by everted sacs of rat small intestine either without or with preincubation with HIFC (Experiments 9, 10 and 11, Table I). This was true when either or both agents were added to the incubation medium just before the start of the second incubation and prior to the addition of vita$\min \mathrm{B}_{12}-\mathrm{Co}^{60}$. The amount of 5,6-dimethylbenzimidazole added was $1 \mu \mathrm{g}$. dissolved in $1 \mathrm{ml}$. of 0.9 per cent $\mathrm{NaCl}$ (producing a ratio of 100 units of 5,6-dimethylbenzimidazole to 1 unit of vitamin $\mathrm{B}_{12}-\mathrm{Co}^{60}$ by weight). The amount of $\mathrm{CoCl}_{2}$ added was $1 \mathrm{ml}$. of $0.11 \mathrm{M}$ solution.

Effect of incubation at $p H$ 1.85. Performance of either the first or the second incubation at $\mathrm{pH} 1.85$ instead of $\mathrm{pH} 7.5$ had no effect on uptake

4 Microscopic preparations made through the courtesy of Dr. H. M. Zimmerman, Department of Pathology, Montefiore Hospital.

5 Kindly provided by Dr. K. Folkers, Merck Sharp \& Dohme Research Laboratories, Rahway, N. J. of control sacs but reduced the enhancement of vitamin $\mathrm{B}_{12}-\mathrm{Co}^{60}$ uptake normally observed after incubation with HIFC (Experiments 12 and 13, Table I).

\section{DISCUSSION}

A previous study (8) demonstrated that HIFC enhanced vitamin $B_{12}$ uptake by open segments of rat small intestine. Since both the mucosal and serosal surfaces were exposed to the incubation medium, such open segments were not suited to study of the mechanism of intrinsic factor action on the mucosal surface; results were complicated by serosal surface vitamin $B_{12}$ uptake which markedly elevated control values. This problem was eliminated in the present study by using closed everted sacs of rat small intestine (6). Only the mucosal surface of such sacs is exposed to the incubation medium.

The results of this study with everted sacs of rat small intestine are strikingly similar to the results of prior studies with rat liver slices (3, 4). In both cases, enhancement of vitamin $\mathrm{B}_{12}-\mathrm{Co}^{80}$ uptake by HIFC is most marked in a sequential incubation system (i.e., a system of incubation with HIFC alone followed by a second incubation with vitamin $\mathrm{B}_{12}-\mathrm{Co}^{60}$ alone). In both cases this enhancement is observable in the cold, is maximal in the presence of adequate calcium, and is reversible to a significant degree by EDTA. In a calcium-free medium, no enhancement of vitamin $B_{12}$ uptake attributable to intrinsic factor occurs on liver slices, but a definite enhancement is still observable with everted sacs of small intestine. This may be due to difficulty in washing the everted sacs free of calcium prior to incubation.

That the increase in the mucosal uptake of vitamin $\mathrm{B}_{12}-\mathrm{Co}^{60}$ after preincubation with HIFC is due to intrinsic factor is suggested by the facts that: 1) the increase is abolished by preheating the HIFC at $100^{\circ} \mathrm{C}$. for 15 minutes, a process which destroys intrinsic factor activity $(9) ; 2$ ) performance of either incubation at $\mathrm{pH} 1.85$ markedly reduces the enhancement of the uptake of vitamin $\mathrm{B}_{12}-\mathrm{Co}^{60}$ otherwise observable (and no enhancement occurs if both incubations are performed at $\mathrm{pH} 1.85[10]$ ). (At this $\mathrm{pH}$ the effect of intrinsic factor in enhancing vitamin $\mathrm{B}_{12}$ absorption from the rat intestine in vivo is 
reduced [11]. It is not yet clear how much of the reduction is due to intrinsic factor instability

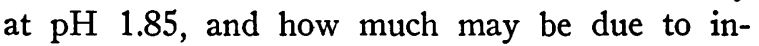
stability of vitamin $\mathrm{B}_{12}$-intrinsic factor or intrinsic factor-receptor bonds at this $\mathrm{pH}$.$) ; 3)$ 5,6-dimethylbenzimidazole, either alone or in combination with $\mathrm{CoCl}_{2}$, does not affect the binding by the system of vitamin $\mathrm{B}_{12}-\mathrm{Co}^{60}$, as it does not interfere with intrinsic factor action in human subjects (12). This latter point may suggest that in the sac formed by the everted small intestine of the rat, as in the small intestine of the human subject, the 5,6-dimethylbenzimidazole portion of cyanocobalamin, as well as the cobalt portion, may not be involved in the interaction with intrinsic factor. Of course, although these substances as such did not interfere with the uptake of vitamin $\mathrm{B}_{12}$, it is possible that as portions of the cyanocobalamin molecule they may be involved in the interaction with intrinsic factor.

These findings would suggest that the mechanism of intrinsic factor action may be the same in the small intestine as in the liver slice. It may be postulated that intrinsic factor has the ability on the one hand to bind vitamin $B_{12}$ (9) and on the other to attach to liver or intestine receptors in the presence of calcium. Possession of these two properties simultaneously may be unique to intrinsic factor. Heparin, chondroitin sulfate and orosomucoid do not increase vitamin $\mathrm{B}_{12}-\mathrm{Co}^{80}$ uptake by everted sacs of rat small intestine (10), as they did not increase vitamin $\mathrm{B}_{12}-\mathrm{Co}^{60}$ uptake by rat liver slices $(3,4)$. This would indicate that enhancement of vitamin $B_{12}$ uptake is not a nonspecific property of any mucopolysaccharide or mucoprotein.

The concept of an intestine receptor for intrinsic factor fits all the facts which led Wallerstein, Harris, Schilling and Castle (13) to "infer that the fundamental action of intrinsic factor is on a superficially located hypothetical structure or chemical process in the wall of the upper small intestine"; and led Glass, Boyd and Stephanson (14) to postulate the existence of an "intestinal vitamin $\mathrm{B}_{12}$ acceptor." In addition, it allows of explanation of the control of absorption of physiologic amounts of vitamin $B_{12}$ in terms of a single mechanism at the cell surface, rather than of separate mechanisms at the mucosal membrane and intramurally.

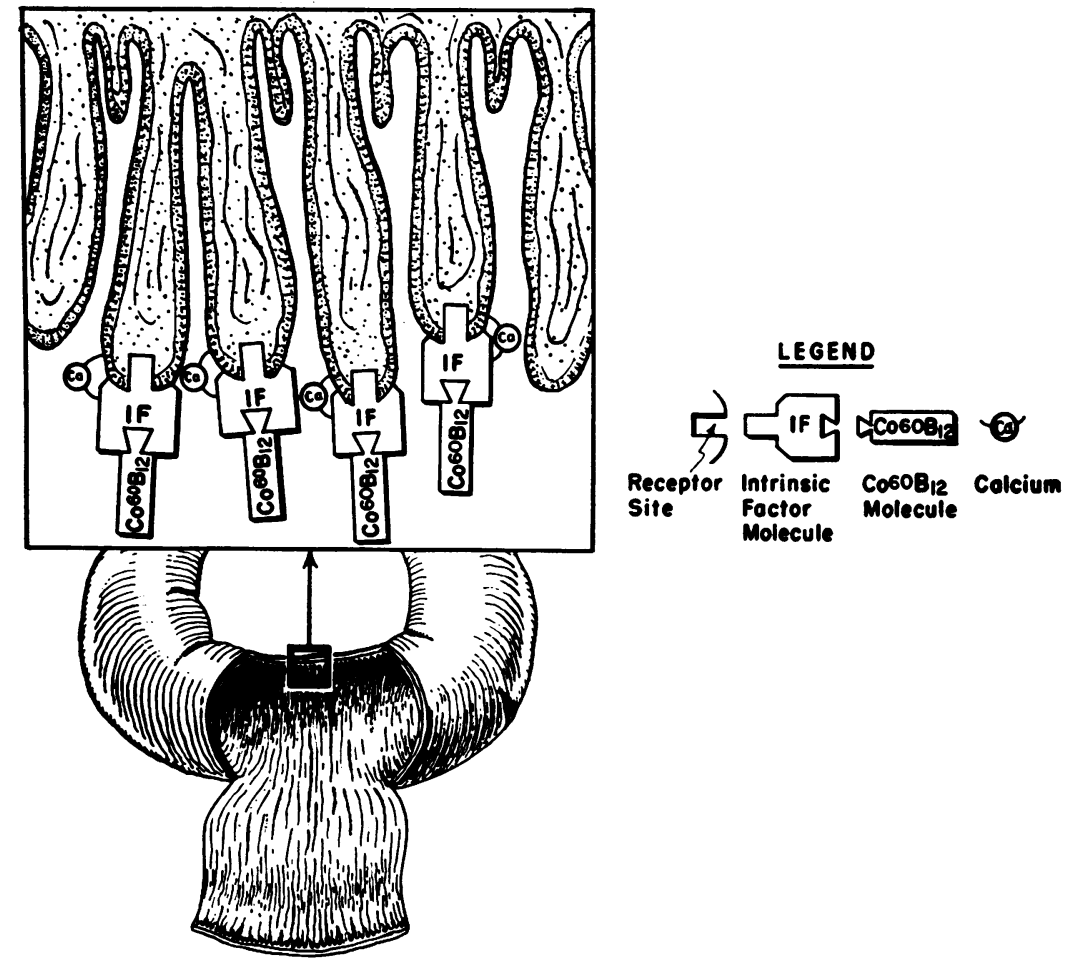

Fig. 1. Optimal Intrinsic Factor Concentration 


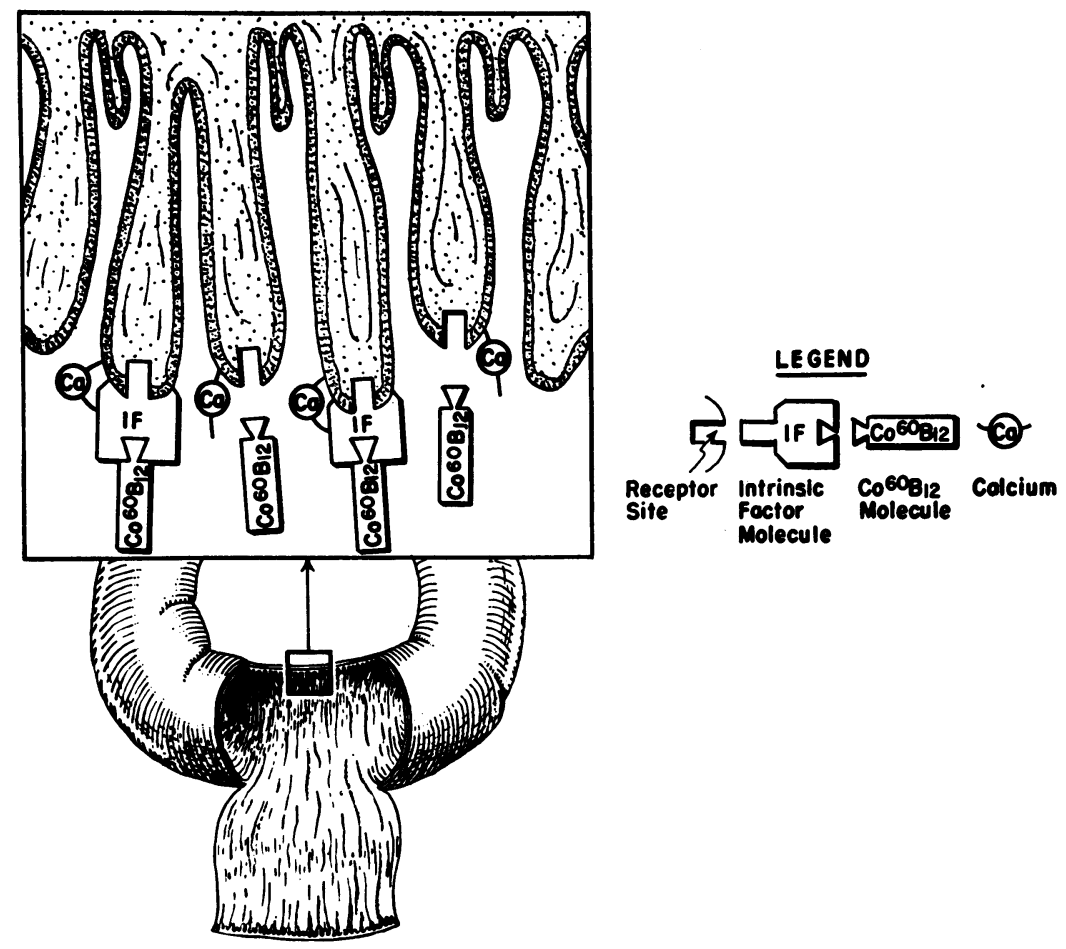

Fig. 2. Half Optimal Intrinsic Factor Concentration

This formulation of the role of intrinsic factor in the first step in the absorption of physiologic amounts of vitamin $B_{12}$ is schematically depicted in Figures 1,2 and 3. Figure 1 pictorially represents the ideal situation: an identical number of receptor sites, intrinsic factor molecules and vitamin $\mathrm{B}_{12}-\mathrm{Co}^{60}$ molecules. Maximal absorption of vitamin $B_{12}$ would occur in this optimal situation. In Figure 2, the decreased uptake of vitamin $\mathrm{B}_{12}$ in the presence of an inadequate amount of intrinsic factor is portrayed. Figure 3 indicates the way the hypothesis of "receptor sites for intrinsic factor" may be used to explain the observed phenomenon in vivo (15-17) of decreased vitamin $\mathrm{B}_{12}$ absorption in the presence of large amounts of intrinsic factor concentrates. When there are more molecules of intrinsic factor than there are receptor sites or molecules of vitamin $\mathrm{B}_{12}$, a portion of the receptor sites will be occupied by intrinsic factor molecules which are not binding vitamin $B_{12}$. Since the excess of intrinsic factor saturates the receptor sites, that vitamin $B_{12}$ which remains in the lumen of the intestine, bound to intrinsic factor which has no available receptor site to go to, is lost in the feces.
It must be emphasized that alternative explanations for the inhibitory effect of large amounts of HIFC must be considered. These include: 1) possible contamination of HIFC preparations with nonintrinsic factor vitamin $\mathrm{B}_{12}$ binding materials; 2) possible presence in HIFC preparations of inhibitors of intrinsic factor action. Neither of these possibilities can be ruled out until intrinsic factor is isolated in pure form. The most potent HIFC preparation available (No. WES655) ${ }^{6}$ inhibited vitamin $B_{12}$ uptake by everted sacs of rat small intestine when used in large quantity (10), but even this preparation can not be considered free of contaminants.

Whether this schema is an accurate depiction of the first stage of vitamin $B_{12}$ absorption in vivo remains to be determined. Preliminary observations, using sodium phytate to "tie up" calcium in the intestine of human subjects, suggest that the mechanism of intrinsic factor action may indeed be calcium-dependent (18). In other stud-

\footnotetext{
${ }^{6}$ Kindly provided by Dr. W. L. Williams and Dr. L. Ellenbogen. This preparation was clinically active in a daily oral dose of $1 \mathrm{mg}$., as determined by urinary excretion studies of the Schilling type (7).
} 
ies of the relationship of results in the in vitro system here reported to those in vivo, it was found that the maximal effect of HIFC occurs in those everted sacs from the middle two quarters of the small intestine (10); the maximal effect of intrinsic factor in the living rat is also in the middle quarters of the small intestine (19). The reason for discarding the proximal $6 \mathrm{~cm}$. of small intestine in all the everted sac experiments reported here was that no effect of HIFC was demonstrable in that portion of the gut.

A further parallel of the in vitro system with the in vivo situation is the demonstration of two separate mechanisms of vitamin $B_{12}$ uptake in both. One mechanism is intrinsic factor-dependent, and plays the major role in absorption of physiologic amounts of vitamin $\mathrm{B}_{12}(20,21)$. It is this mechanism with which this report is mainly concerned. The other mechanism appears to be a "mass action phenomenon," possibly diffusion, and is operative in bringing about the absorption of small amounts of vitamin $B_{12}$. This latter mechanism is most readily observable in the human subject after the oral administration of supraphysiologic amounts of vitamin $B_{12}$
$(20,21)$. In the everted sac of rat small intestine, it may be this mechanism which produces the vitamin $B_{12}$ uptake observed in controls.

The failure to observe enhanced vitamin $\mathrm{B}_{12}-\mathrm{Co}^{60}$ uptake by everted sacs of rat small intestine after incubation with large amounts of HIFC, or after simultaneous incubation with amounts of HIFC which produced marked enhancement of liver slice vitamin $\mathrm{B}_{12}$ uptake, is not yet explained. The former may be explainable by "trapping" of large amounts of HIFC between the intestinal villi, to be released during the subsequent incubation with vitamin $\mathrm{B}_{12}-\mathrm{Co}^{80}$ and then to compete for the vitamin with the intrinsic factor on receptor sites. The latter may be explainable by the presence of fewer free receptors on the intestinal wall than on the liver slice. This is suggested by the fact that enhancement of vitamin $B_{12}$ uptake by HIFC is not as many times more than control values for intestine sacs as for liver slices. It is possible that many receptor sites on the rat irtestinal wall are occupied by rat intrinsic factor binding vitamin $\mathrm{B}_{12}$ taken in the rat's diet prior to sacrifice. Studies with everted sacs of small intestine from

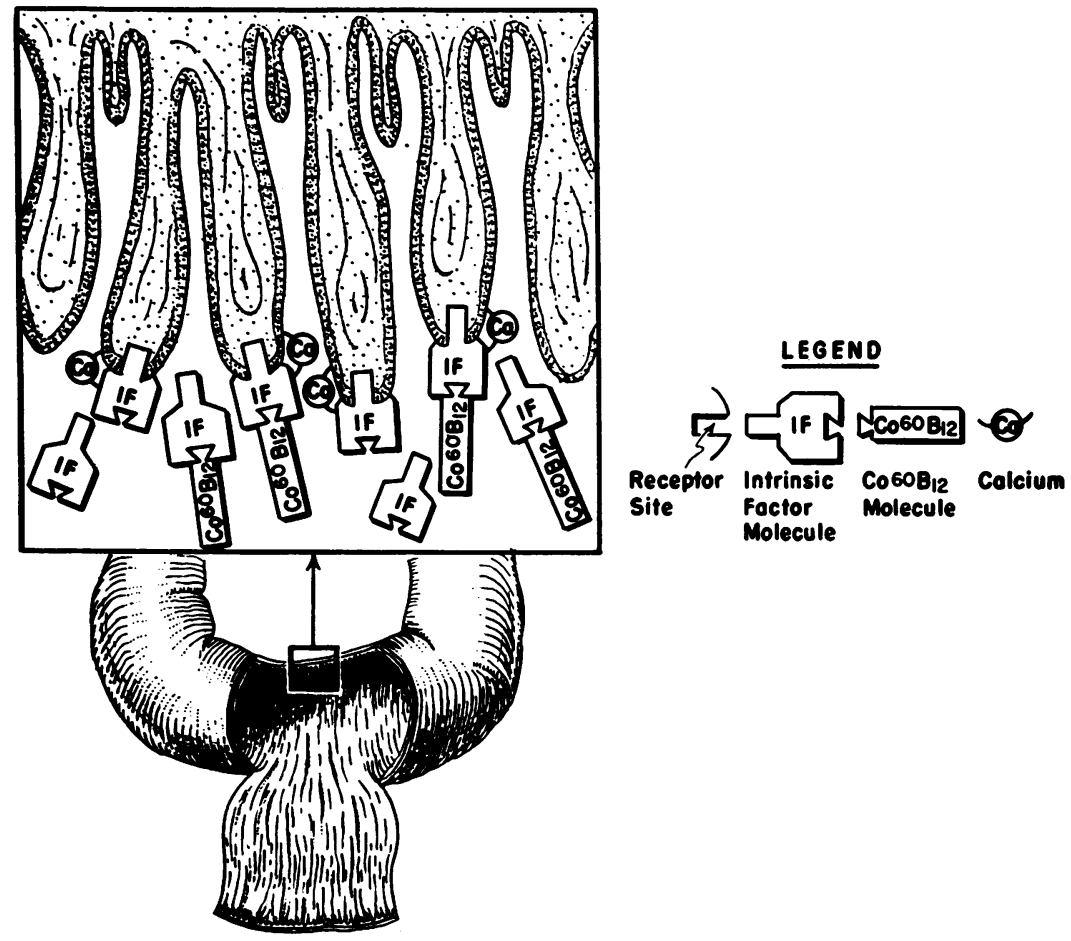

Fig. 3. Twice Optimal Intrinsic Factor Concentration 
gastrectomized rats may provide useful information in this connection.

Finally, the demonstration that hog intrinsic factor concentrate will enhance vitamin $B_{12}$ uptake by rat small intestine further weakens the indirectly (22) and directly (17) attacked concept of species specificity of intrinsic factor as a phenomenon of major importance. Data previously reported (8) would suggest the interesting speculation that perhaps an organ specificity may exist for intrinsic factor and may be of greater significance than the species specificity.

\section{SUM MARY}

Vitamin $B_{12}$ uptake by everted sacs of rat small intestine is enhanced markedly after preincubation with hog intrinsic factor concentrate (HIFC). This enhancement occurs at $6^{\circ} \mathrm{C}$., is maximal in a calcium-containing incubation medium, and is reversible to a significant degree by disodium ethylenediaminetetraacetate dihydrate (EDTA). Procedures which interfere with intrinsic factor action, such as heating at $100^{\circ} \mathrm{C}$. or incubating at $\mathrm{pH} 1.85$, eliminate or reduce this enhancement.

The close similarity of these findings to those previously obtained with rat liver slices is discussed. The hypothesis is presented that the first step in the absorption of physiologic amounts of vitamin $B_{12}$ by the small intestine is binding of the vitamin to intrinsic factor and binding of intrinsic factor to intestine receptors (in the presence of calcium). It is suggested that possession of these two binding properties simultaneously may be unique to intrinsic factor.

The uptake of vitamin $\mathrm{B}_{12}$ by everted sacs of rat small intestine in the absence of HIFC may be the in vitro parallel of a nonintrinsic factordependent mechanism of vitamin $\mathrm{B}_{12}$ absorption observed in vivo and operative primarily in the presence of supraphysiologic amounts of vitamin $\mathrm{B}_{12}$.

\section{ACKNOWLEDGMENT}

The author is indebted to Dr. Theodore $\mathrm{H}$. Spaet for his advice and help throughout this study and in the preparation of the manuscript.

\section{REFERENCES}

1. Castle, W. B. Medical progress; development of knowledge concerning the gastric intrinsic factor and its relation to pernicious anemia. New Engl. J. Med. 1953, 249, 603.

2. Heathcote, J. G., and Mooney, F. S. The oral treatment of pernicious anaemia. Lancet 1958, 1, 982.

3. Herbert, V. Studies of the mechanism of the effect of hog intrinsic factor concentrate on the uptake of vitamin $B_{12}$ by rat liver slices. J. clin. Invest. 1958, 37, 646.

4. Herbert, V. Development of a possible in vitro assay for intrinsic factor. Proc. Soc. exp. Biol. (N. Y.) 1958, 97, 668.

5. Herbert, V. Mechanism of intrinsic factor action in the isolated rat small intestine (abstract). J. clin. Invest. 1958, 37, 901.

6. Wilson, T. H., and Wiseman, G. The use of sacs of everted small intestine for the study of the transference of substances from the mucosal to the serosal surface. J. Physiol. 1954, 123, 116.

7. Ellenbogen, L., Williams, W. L., Rabiner, S. F., and Lichtman, H. C. An improved urinary excretion test as an assay for intrinsic factor. Proc. Soc. exp. Biol. (N. Y.) 1955, 89, 357.

8. Herbert, V., and Spaet, T. H. Distribution of "intrinsic factor" activity. Amer. J. Physiol. 1958, 195, 194.

9. Grasbeck, R. Studies on the vitamin $B_{12}$-binding principle and other biocolloids of human gastric juice. Acta med. scand. 1956, Suppl. 314.

10. Herbert, V. Unpublished observations.

11. Niewig, H. O., Shen, S. C., and Castle, W. B. Mechanism of intrinsic factor action in the gastrectomized rat. Proc. Soc. exp. Biol. (N. Y.) 1957, 94, 223.

12. Bunge, M. B., Schloesser, L. L., and Schilling, R. F. Intrinsic factor studies. IV. Selective absorption and binding of cyanocobalamin by gastric juice in the presence of excess pseudovitamin $B_{12}$ or 5,6dimethylbenzimidazole. J. Lab. clin. Med. 1956, 48, 735 .

13. Wallerstein, R. O., Harris, J. W., Schilling, R. F., and Castle, W. B. Observations on the etiologic relationship of achylia gastrica to pernicious anemia. XV. Hematopoietic effects of simultaneous intravenous and of simultaneous or serial oral administration of intrinsic factor and vitamin $B_{12}$. J. Lab. clin. Med. 1953, 41, 363.

14. Glass, G. B. J., Boyd, L. J., and Stephanson, L. Intestinal absorption of vitamin $B_{12}$ in humans as studied by isotope technic. Proc. Soc. exp. Biol. (N. Y.) 1954, 86, 522.

15. Glass, G. B. J., Boyd, L. J., Stephanson, L., and Jones, E. L. Metabolic interrelations between intrinsic factor and vit. $B_{12}$. III. $B_{12}$ absorption at varied intrinsic factor doses. Proc. Soc. exp. Biol. (N. Y.) 1955, 88, 1.

16. Callender, S. T., and Evans, J. R. Observations on the relationship of intrinsic factor to the absorption of labelled vitamin $B_{12}$ from the intestine. Clin. Sci. 1955, 14, 387.

17. Taylor, K. B., Mallett, B. J., Witts, L. J., and Taylor, 
W. H. Observations on vitamin $B_{12}$ absorption in the rat. Brit. J. Haemat. 1958, 4, 63.

18. Herbert, V. Studies of intrinsic factor mechanism of action. Vitamin $B_{12}$ Symposium, New York Medical College, April 11, 1958. In press.

19. Reynell, P. C., Spray, G. H., and Taylor, K. B. The site of absorption of vitamin $B_{12}$ in the rat. Clin. Sci. 1957, 16, 663.

20. Ross, G. I. M., Mollin, D. L., Cox, E. V., and Ungley, C. C. Hematologic responses and concentration of vitamin $B_{12}$ in serum and urine following oral administration of vitamin $B_{12}$ without intrinsic factor. Blood 1954, 9, 473.

21. Doscherholmen, A., and Hagen, P. S. A dual mechanism of vitamin $B_{12}$ plasma absorption. J. clin. Invest. 1957, 36, 1551.

22. Abels, J., Woldring, M. G., Vegter, J. J. M., and Niewig, H. O. Effect of rat intrinsic factor on vitamin $B_{12}$ absorption in pernicious anemia. Science $1957,126,558$. 